\title{
Training and supporting pharmacists to supply progestogen-only emergency contraception
}

\author{
Lesley Bacon, MFFP, MRCGP, Consultant in Reproductive Health, Community Health South London NHS Trust; \\ Imogen Savage, PhD, MRPharmS, Lecturer in Primary Care Pharmacy, King's College London; Sue Cook, BPharm, MRPharmS, \\ Project Manager, Community Services Pharmacy Team, Community Health South London NHS Trust; Beth Taylor, BSc, \\ MRPharmS, Manager, Community Services Pharmacy Team, Community Health South London NHS Trust, London, UK
}

Correspondence: Dr L Bacon, Department of Reproductive Health, Lewisham Primary Care Trust, St Giles, St Giles Road, London SE5 7RN, UK. Tel: +44 (0) 207771 3330.E-mail:mikembacon@aol.com

(Accepted 2nd February 2003)

Journal of Family Planning and Reproductive Health Care 2003; 29(2): 17-22

\begin{abstract}
Objective. To describe and evaluate the training and support provided to the first cohort of community pharmacists to supply progestogen-only emergency contraception (POEC) under a Patient Group Direction $(P G D)$ in Lambeth, Southwark and Lewisham, London.

Design. The study comprised (a) a systematic analysis of written and oral data from pharmacists before and during training, and at 5 and 13-14 months after launch; (b) analysis of telephone calls to clinical support and (c) analysis of written pharmacy records.

Subjects. A total of 20/22 pharmacists in the first training cohort; $6 / 23$ pharmacists who applied but were not accepted were also followed up.

Results. A formal course with role-play was a successful training method, and the course also served as a teambuilding exercise. Subsequent interviews demonstrated that pharmacists had understood the concept of client confidentiality and gained confidence over time in the use of the PGD. The on-call consultants received 152 calls in the first 12 months of the scheme. Over $80 \%$ of the calls concerned clinical criteria (notably including 22\% that were queries about oral contraceptives). Frequency ranged from one to eight calls per week with $28 \%$ made at weekends. In over half (60\%) of the calls the pharmacist was subsequently able to make a supply. Queries over client management resulted in several changes in the protocol. The primary expressed concern for all pharmacists at all time points was how clients might 'misuse' or 'abuse' the service, and this remained a concern despite the fact that it also applies to other routes of supply of POEC. However, the PGD cohort was more positive on local benefits than pharmacists who were not selected.

Conclusions. Training and support have enabled this often-underused group of professionals to participate in an extended reproductive health service. Mobile phones are an essential support tool.
\end{abstract}

\section{Key message points}

- Pharmacists can be trained to provide progestogen-only emergency contraception and to become part of an integrated reproductive health care system.

- The course can be used to build strong links with local pharmacists. Although 'high street' pharmacists know their local general practitioners well, they may not be familiar with community reproductive health services.

- After training, round-the-clock access to clinical support via mobile phone helps pharmacists to work confidently within the Patient Group Direction (PGD), and provides useful feedback on training needs and on points in the PGD that may need to be reviewed.

- Training and support enables a group of professionals whose training is often underused to participate in a seamless service.

\section{Introduction}

The need to improve access to emergency hormonal contraception (EHC) is widely recognised. ${ }^{1,2}$ Research in the UK and elsewhere has confirmed that women who need EHC can find it hard to see a doctor within the 72-hour time window because surgeries and clinics are not always open when women need them. ${ }^{3}$ The UK is well-supplied with community pharmacies. Most are open 6 days a week, and their hours tend to be longer than those of surgeries or reproductive health clinics. ${ }^{4}$ [All the pharmacies selected for this pilot were open on Saturday, and four were open on Sunday, whereas there were only two family planning clinics (FPCs) in Lambeth, Southwark and Lewisham on a Saturday.] Women tend to visit pharmacies more than men, and for a wider range of reasons. ${ }^{5}$ Pharmacies therefore offer an 'no appointment' pathway to EHC which might be attractive to young women.

In 1998, the Lambeth, Southwark and Lewisham (LSL) Health Authority area became a Health Action Zone (HAZ). LSL has high levels of deprivation, and the teenage pregnancy rate is one of the highest in the country. (In 2000 it was 79.7 per 1000 women aged 15-17 years, compared with 43.8 for the whole of England and Wales.) ${ }^{6}$ Reducing underage pregnancy was a key aim in the HAZ programme of work, and the need to widen access to emergency contraception was generally accepted. The Local Pharmaceutical Committee (LPC) suggested a pilot project in which community pharmacists would supply oral progestogen-only emergency contraception (POEC) under a Patient Group Direction (PGD). Funding was obtained, and the project was developed as a partnership between the HAZ, the LPC and Community Health South London NHS Trust, which undertook the project management. In January 2000, all 180 pharmacy contractors in LSL were invited to apply for the HAZ pilot scheme and 45 applied to join. Twenty-two pharmacists were selected using the criteria described below and the pilot service was launched in April 2000 through 20 accredited community pharmacies.

The selection criteria were:

- Prior health promotion training

- Suitable premises (including a quiet area where clients could talk privately)

- Availability in the pharmacy at least 4 days a week (including Saturday and/or Sunday)

- Enthusiasm for the project

- Geographical location.

Documented evidence of prior health promotion training was the primary criterion. The majority of pharmacists rejected for the first training round either had not actually done such training, or could not provide evidence of it. 
Box 1: Itinerary for the 3-day course

Day 1

- Introduction to the scheme (including the Manchester pilot)

- Clinical aspects of emergency contraception (oral and intrauterine)

- Medico-legal and ethical aspects of emergency contraception

- Review of hormonal methods of contraception

- Working with the under-16s (including Fraser guidelines)

Day 2

- Working with protocols

- Dealing with sexual assault and the distressed client

- History taking

- Referral (for continuing contraception) and follow up

- Cultural and religious aspects

Day 3

- Intrauterine devices

- Barrier methods

- Role play with professional role players

- Clinical and managerial support

- Pregnancy testing and review

An information pack (copies available from the authors) was provided after the course.

Role-play (angry parent; frightened teenager; undercover reporter: scenarios available from the authors) was included to cover situations which pharmacists had indicated might concern them. The usual ground rules for role-play were explained. [The pharmacists were used to dealing with angry customers and difficult situations (e.g. some were involved in the local needle exchange programme). However, the project team was very conscious that the pharmacists would be working in isolation, and that any adverse publicity might affect the rest of their business.]

To supply POEC under a PGD, both the pharmacist and the pharmacy in which he or she practises must be accredited to make a free National Health Service (NHS) supply. All selected pharmacies were inspected by a member of the project team, who assessed both physical facilities and the pharmacist's attitude and working methods.

Pharmacists became accredited by completing a training programme (see Box 1) devised by the South East London Family Planning Training Unit. The first 22 pharmacists attended a 3-day course, 1 day a fortnight over a period of 6 weeks. They then signed an 'approved practitioner' statement agreeing to act within the terms of the PGD.

Two 'cascade' support systems were developed: one for clinical support and one for operational matters. The clinical support system relied on mobile phones with voice mail facilities and involved all four local reproductive health consultants. Operational (professional and administrative) support was provided by a pharmacist project manager (S.C.).

The training that these PGD pharmacists received is not the same as that devised for pharmacists selling POEC (as Levonelle-2), which became legal in January 2001. The aim of the HAZ programme was to stress the involvement of accredited pharmacists in health education and referral to other sources of ongoing care.

\section{Methods}

\section{Evaluation of the training course}

Information from evaluation sheets completed at the end of the course was included in the qualitative analysis.

\section{Evaluation of the effects of training and support on pharmacists}

Three primary data sources were used as detailed below.

Application forms. These provided written verbatim comments from all pharmacists on initial concerns about the scheme before training, and on perceived benefits to them and their local community.
Interviews. These were conducted with 20/22 PGD pharmacists, using a topic guide based on the issues and themes generated by six pharmacists in a group discussion held soon after the launch of the scheme. Interviews were done 5 months after launch [when POEC was still a prescription-only medicine (POM)] and 13-14 months after launch (4-5 months after deregulation). Six pharmacists of the 23 who applied but were unsuccessful were also followed up; five in a group discussion and one with an interview. Interviews were usually carried out in the pharmacy; group discussions took place at King's College London. They were tape-recorded, transcribed and analysed by one of us (I.S.), who had no involvement with training or support. The thematic framework used was validated by an independent researcher from another university.

All the data from interviews, group discussions and written comments on application forms were included in the qualitative analysis. This used the Framework method ${ }^{7}$ in which a thematic framework, or detailed index, is drawn up and applied systematically to all textual data. Data is then rearranged, or charted, for each key theme, respondent by respondent. This allowed the number of pharmacists mentioning specific issues at any given time point to be counted. NB. Text in italic in quotation marks indicates the speaker's own words.

Clinical support log. The vast majority of calls from pharmacists were dealt with by L.B., who maintained a written record of the nature and outcome of each query. These records were coded by I.S. and verified by L.B. The clinical categories were those specified in the PGD. The outcome of each call (supply POEC, refer, no need for POEC) was also determined. The Statistical Package for the Social Sciences (SSPS, Chicago, IL, USA) was used for analysis.

\section{Triangulation}

Pharmacist interviews and the clinical support call log provided two different, but complementary, perspectives on how the POEC scheme was operating. Anonymous audit data, ${ }^{8}$ completed by clients in pharmacies at the time of supply, provided independent verification of pharmacist accounts of workload, client demography and contraceptive history and the proportion of cases needing onward referral and/or clinical support. A third perspective of pharmacist training and support issues was obtained though interviews with four members of the project team.

\section{Results}

\section{Evaluation of the training course}

Speakers at the 3-day training course came from a range of health and social care professions. Comments on the postcourse evaluation sheets showed that the pharmacists liked this detailed training but inevitably there were minor disagreements on the content balance. Some picked up completely new facts while others thought that some of the medicine-related information was really revision, and could be read before the course to save time.

Teaching on condoms and pregnancy testing was said to have been superfluous, but information about other methods such as intrauterine devices (IUDs) was useful, as most had never seen an IUD out of its packet. Information on other local services was also valuable. Their local general practitioners (GPs) were well known to the pharmacists, but local community and genitourinary medicine (GUM) services were not. At the time of training (early 2000) the role of the 24-hour telephone helpline, NHS Direct, was also unfamiliar. 


\begin{tabular}{|c|c|c|c|c|}
\hline \multirow[t]{2}{*}{ Issues/concerns } & \multicolumn{4}{|c|}{ Pharmacists mentioning issue (n) } \\
\hline & Pre $(N S)^{\mathrm{a}}$ & Pre $^{b}$ & Earlyc & Late $^{\mathrm{d}}$ \\
\hline \multicolumn{5}{|l|}{ How service might be used } \\
\hline Misuse as contraceptive & 5 & 8 & 8 & 7 \\
\hline Abuse of the supply system & 8 & 3 & 6 & 4 \\
\hline \multicolumn{5}{|l|}{ Supplying service under a PGD } \\
\hline Deciding if client falls in protocol & 5 & 2 & 9 & 4 \\
\hline Managing clients outside protocol & - & 2 & 4 & - \\
\hline Providing safe sex education & - & 2 & 2 & - \\
\hline Liability; confidentiality (under-16s); local reaction & 5 & 4 & 2 & 3 \\
\hline \multicolumn{5}{|l|}{ Operational factors } \\
\hline Training, support, time, paperwork & 2 & 4 & - & - \\
\hline Locum cover & - & - & 2 & 2 \\
\hline Impact of deregulation & & & & 9 \\
\hline
\end{tabular}

aMentioned in pre-training assessment by pharmacists who were not selected for the programme.

bMentioned in pre-training assessment by pharmacists who were selected for the programme.

cMentioned 5 months after starting to supply.

dMentioned 14 months after starting to supply.

NS, not selected; PGD, Patient Group Direction.

Role-play was a new way of working for the participants, and proved to be particularly useful. In the light of experience, they recognised that the role-play situations used illustrated extremes, but were unanimous in support of their value in terms of addressing pre-scheme anxieties. However, they also thought it would be good to add a straightforward case: 'to prepare for what's likely to happen'. They also requested more time for education on the reproductive cycle.

It quickly became apparent that the courses also served as a team-building exercise, enabling the participants, the project manager and community trust pharmacists, and the clinicians who would be offering support to get to know each other and work as an effective unit.

\section{Evaluation of concerns about supplying EHC}

Table 1 shows the concerns expressed by pharmacists who applied to participate in the scheme. The pharmacists described in this paper were a highly selected group, representing half of all applicants, but only a small proportion (approximately 15\%) of all the pharmacies in LSL. The majority (15) were male pharmacy contractors (owners) who had worked in the area for many years.

The pattern in pharmacists who were and were not selected was broadly similar. Pharmacists' primary concern before (and during) the pilot scheme was how the service might be used by clients. Pharmacists used two terms here: misuse of POEC as a contraceptive and abuse of the system for supplying it through pharmacies.

The first pharmacist cohort was highly motivated to provide a public health service which in the words of one contractor was 'begging to be done'. They saw a clear need for the new service in their own area, and were more likely to mention specific benefits to their local community on their application form. Pharmacists who were not selected tended to put professional satisfaction and/or personal development first.

The issues of legality, liability, and supply to under-16s did not feature particularly highly in pre-training comments. However, by the time selected pharmacists reached the first training day they had had time to reflect on what taking part in the scheme really meant. At the time, the concept of supplying a POM from a pharmacy under a PGD was new and still quite controversial. The training helped all of them to acknowledge their fears over their legal position, bad publicity and being 'mystery shopped'.
Changes in needs and concerns over time

Issues and concerns expressed in interviews with pharmacists (Table 1) suggested that the pharmacists gained confidence in decision-making as time went on. Queries over client management within the PGD resulted in several changes in the protocol and use of the support service then decreased.

In contrast, anxieties in the six non-PGD pharmacists interviewed increased across the board as they gleaned second-hand information. They were unsure how the PGD worked, confused about the time required, and bothered about local reaction. One felt the scheme was 'creating classes of pharmacists'.

Both supplying and non-PGD pharmacists raised concerns about how the service might be used. Misuse was usually spoken of in terms of safety (e.g. using 'too often') or efficacy (keeping POEC 'just in case'.) Abuse covered the concept of clients learning to manipulate the supply system, e.g. by obtaining supplies from several pharmacies, and requests made by a man on a woman's behalf.

Impact of deregulation. From pharmacist accounts there was little awareness, and little reaction, from customers or local people before POEC became available over the counter (OTC), although one non-PGD pharmacist described gaining two customers (who disapproved of the scheme) from a PGD pharmacy.

However, deregulation of POEC received considerable media coverage in the latter part of 2000 , culminating in a (factually incorrect) news story in the Daily Mail on 6 January 2001. The item ${ }^{9}$ named seven PGD pharmacists and claimed that six had 'broken the rules' by supplying a 15-year-old girl. The pharmacists had been trained for just this eventuality, and those targeted coped well, but the incident made the whole group very angry and some lost their confidence. This was reflected in the number of 'no need' calls to the support clinician, which increased from $5 \%$ of calls in the period October-December 2000 to $18 \%$ of all calls between January and March 2001. Pharmacists who had not previously used the service also called for advice during this period. This loss of confidence was relatively short-lived.

As Table 1 shows, the OTC move was a significant issue for the PGD pharmacists. While client cost was a factor, their major expressed concern was the difference in pharmacist training and support. The confidential records 
that they made demonstrated that the pharmacist had acted in good faith on what the client had said, and would protect them in the event of a claim for negligence. With OTC supply there was no register of pharmacist training, no record keeping, and no accountability.

Confidentiality. Training placed great emphasis on the importance of confidentiality and subsequent interviews provided evidence that the first cohort had taken this message on board. The pharmacists sometimes referred to the written client records that they were required to keep, but were careful not to show these to the interviewer (I.S.). The pharmacists did not consider that there should be a mechanism for informing a client's GP that supply had been made. The only time they would pass information on would be with a referral form, which the client could choose to pass on.

\section{Evaluation of the clinical support given to the pharmacists} Demand for the scheme was greater than expected, with 6294 women accessing the scheme in the first year. The vast majority of consultations were straightforward; less than $6 \%$ of clients did not meet the requirements for supply under PGD. ${ }^{8}$ In cases where the pharmacist was uncertain, clinical support often helped them to decide. In the first 12 months of the scheme 152 telephone calls were made and logged; for 150 calls the outcome was clear. For more than half the calls the pharmacist was able to make a supply after advice from the consultant.

Call frequency ranged from one to eight calls per week, with an average of around 13 calls per month. Over a quarter $(28 \%)$ of all calls were made at weekends.

Table 2 shows the type of queries received, and the outcome. In two cases this was conditional on the results of a pregnancy test, and in two cases on further assessment of the woman's menstrual cycle.

Over $80 \%$ of all calls related to specific clinical criteria set down in the PGD. These were:

- Date of last menstrual period (LMP)

- Cycle length

- Length of LMP (i.e. was it normal)

- Possibility of existing pregnancy

- Date and time of unprotected sexual intercourse

- Any other unprotected sexual intercourse this cycle

- Other medication.

The most common difficulty pharmacists had was in determining the date of the LMP. The main problems recounted in early interviews were situations that had not been specifically covered in the original protocol. They were:

- Last dose of injectable contraception some time ago; periods not yet returned

- Breastfeeding after childbirth; periods not returned

- After a termination of pregnancy (described as ' $a$ nightmare' or 'most tricky case').

There were also a number of queries relating to the timing of unprotected sexual intercourse, and estimating the risk of pregnancy. Many of these calls were for reassurance as this was work that was very new to the pharmacists. A female pharmacist noted that a proportion of women did not know their own cycles, nor understand how their bodies worked, and requested educational materials to help her teach them.

The second most common query concerned missed 'pills' and pill-drug interactions, which accounted for a further $22 \%$ of calls. Just under half of these queries came from one pharmacist, with a second accounting for a further eight calls. Eight more pharmacists called at least once about this topic. Confidence in dealing with this problem varied greatly between pharmacists.

The overall frequency of requests for support varied markedly between pharmacists, ranging from less than $1 \%$ to $13.6 \%$ of supplies made. Some of this variation will reflect client differences, but pharmacist confidence is also an important factor. Four pharmacists did not call clinical support at all in the first year of the project and a further three pharmacists made very few calls. Several pharmacists in this low-caller group had good contacts with local surgeries and may have preferred to use a GP colleague as an information source.

Some pharmacists found it easier to make decisions than others. One low-caller said: 'It's pretty black and white with the protocol'. Another explained: 'If I'm stuck I ring up another pharmacist on the course, or they ring me. There are two or three of us, we talk over the phone. If there's the slightest doubt I refer. It's better than taking risks'. There was also an understandable desire to try and resolve uncertainties by talking cases over with fellow PGD pharmacists first: 'I'll think I'll look really stupid if I ask her this. It avoids wasting her time'.

Clinical support was highly valued by all the pharmacists, even those who were infrequent users. The experience was very different from ringing the surgery, where it was hard to get to speak to one of the doctors and a query could take hours to resolve.

Table 2 Pharmacists' clinical queries and their outcome

\begin{tabular}{|c|c|c|c|c|c|}
\hline \multirow[t]{2}{*}{ Query type } & \multicolumn{5}{|l|}{ Outcome } \\
\hline & Conditional & $\begin{array}{l}\text { No need } \\
\text { for } \mathrm{EHC}\end{array}$ & Refer & Supply & Total $(\%)$ \\
\hline Date of LMP & 1 & 3 & 19 & 22 & $45(30)$ \\
\hline Oral contraceptives & 1 & 11 & 1 & 20 & $33(22)$ \\
\hline Unprotected sexual intercourse timing/pregnancy risk & 2 & 3 & 2 & 6 & $13(8.7)$ \\
\hline Interacting medication & & & 3 & 8 & $11(7.3)$ \\
\hline Adverse reactions/contraindications & & & 1 & 10 & $11(7.3)$ \\
\hline Second dose timing & & & 2 & 6 & $8(5.3)$ \\
\hline Cycle character & & & 3 & 4 & $7(4.7)$ \\
\hline Previous emergency contraception this cycle & & & 4 & & $4(2.7)$ \\
\hline \multicolumn{6}{|l|}{ Supply issues (e.g. women from outside the Health Authority } \\
\hline area; third-party supply) & & & & 8 & $8(5.3)$ \\
\hline Locating a clinic for future contraception & & & & 2 & $2(1.3)$ \\
\hline Sexual abuse & & & & 1 & $1(0.7)$ \\
\hline Unclear/multiple & & 1 & 2 & 4 & $7(4.7)$ \\
\hline Totals & 4 & 18 & 36 & 91 & 150 \\
\hline
\end{tabular}

EHC, emergency hormonal contraception; LMP, last menstrual period. 
One high user described it as: 'A personal direct line. Brilliant, really nice. It's what made the project really successful. Initially I felt I needed confirmation of a decision. It's still nice to have her say it's $O K$ '.

Onward referral. Referring clients on to clinics and contraceptive services was a key part of the service. As one project team member explained: 'It's the encouraging to be part of a seamless service we want, which is vital. The point we're trying to make is this is actually a conduit for women to come in and seek medical advice. To bring them into the system'.

From the anonymous client audit data, pharmacists' main onward referral path for contraceptive advice after POEC was to a local FPC (including Brook for under-25s) or the client's GP. The introduction of a referral form in late 2000 was welcomed as giving pharmacists a 'stronger role' in persuading clients to take up their advice and go to a clinic.

For more difficult cases, the clinician log shows referral to 11 specific clinics across LSL.

Pharmacists were supplied with information on clinic locations and opening hours during training. However, some pharmacists later described having difficulty in finding a local clinic which was open and two called clinical support for help over this.

From pharmacist accounts, the place of referral often depended on the day, time and where the woman came from. Some pharmacies with clinics nearby could send women 'round the corner'; for others, the GP was often closer. Local accident and emergency departments were more of a fall-back for out-of-hours situations.

\section{Operational support}

Regular personal contact, by phone and in meetings, was established from the start of the project. The project manager acted as a link between the pharmacists and the project team, and facilitated joint meetings at which issues and concerns could be aired and addressed.

The benefits of this approach were demonstrated at the pilot service's only critical point: the publication of the Daily Mail article. For a short time afterwards pharmacists felt unsettled and nervous about supply to young people. This resolved quite quickly after a meeting at which they could voice their anxieties.

The pharmacists welcomed the chance to get together, and described the meetings as 'rewarding'. They valued the fact that their feedback on grey areas within the PGD were taken up, with a revised PGD produced in early 2001. It was also good to have their training suggestions adopted in subsequent recruitment rounds.

\section{Discussion}

An initial cohort of 22 pharmacists was selected and trained to provide POEC to a PGD. There have since been two further rounds of selection and training, and 36 pharmacies and 51 pharmacists are now able to supply. ${ }^{8}$

When pharmacists applied to join the scheme everyone had much the same anxieties. These were broadly similar to those reported in previous surveys 10 and from the Manchester pilot. ${ }^{11}$ For PGD pharmacists, the majority of these anxieties were addressed in training and in the ongoing support which was provided. Five months into the project, pharmacists were not worried about the operational aspects of the service, although they were starting to think about the need for training locums so continuity of cover could be ensured. Their main concerns were in deciding if supply was appropriate and managing out-of-protocol clients, and for this they found the clinical support invaluable. Community and GUM services which, as others have also found ${ }^{12}$, were unfamiliar to pharmacists were becoming used as part of a seamless service.

In some respects our pharmacists' experience may have been slightly different from those in the Manchester scheme. In Manchester, some pharmacists who had been trained and were currently providing EC under PGD were hesitant about their knowledge base. ${ }^{13}$ This was not apparent in our group, although concern over possible misuse was a shared theme. (This may be insuperable; similar concerns apply in community FPCs where women may travel from one clinic to another as well as seeing their GPs, and may use assumed names and addresses. The only remedy may be education about the danger of failure of the method if it is used instead of a regular method. These pharmacists, unlike a national sample of pharmacists surveyed a year earlier, ${ }^{14}$ did not consider that the woman's GP should be informed of her treatment even though this might be seen as a way of regulating use of EHC.)

At the 15 month point, LSL pharmacists had few concerns around making a supply themselves, although anxieties about abuse of the system remained. They felt well supported and the service had become part of their normal practice.

Providing the back-up service required very little time (about 2 minutes per call) but did require the availability of a mobile phone with voice mail and a willingness to be available. Trusts will need to see mobile phones as being an essential tool for supporting junior staff and nurse-led clinics, as well as pharmacists, in the future.

The pharmacists greatly valued having rapid access to expert clinical advice. This was not something they had experienced in primary care previously. A strong advantage of the training course was that it allowed clinicians and pharmacists to get to know each other and understand more about respective spheres of practice.

The experiences of pharmacists and trainers were used to produce a more time-efficient course, run over 2 days, for subsequent pharmacist cohorts.

The role-play was also altered, with a 'normal' straightforward consultation included to give the participants an insight into the practicalities of supplying. Problems with the media had not, at that point, materialised. However, refusing to supply to clients who did not fit the protocol had been more difficult than had been anticipated, and a scenario was devised to cover this. This situation caused the only incident of actual or threatened violence that we have had so far in the project.

\section{Conclusions}

Training pharmacists to supply oral EC to a PGD proved fulfilling for the trainees and trainers alike. By the end of the first training wave it became apparent that the courses also served as a team-building exercise, enabling the participants, the project manager and community trust pharmacists, and the clinicians who would be offering support to get to know each other and work as an effective unit.

Working with an entirely new group of professionals, who have had a very different basic training, was challenging, and it was necessary to be flexible and to adapt the course in the light of experience. In particular, the availability of clinical and managerial helplines after the project went 'live' revealed issues that needed to be tackled in the second wave of training and in reviewing and revising the PGD.

The project concerned only NHS supply, and was not related to the POM-to-P switch. However, the course may well be relevant to the latter. It may also be relevant to other work to PGDs that pharmacists may undertake in the future. 
At a time when primary health care resources are increasingly stretched, training and ongoing support are enabling this often underused group of professionals to participate in an extended reproductive health service to women in LSL.

\section{Acknowledgements}

The project team would like to thank everyone who has been instrumental in the project's success.

\section{Statements on funding and competing interests}

Funding. This project and its evaluation were funded by Lambeth Southwark and Lewisham Health Action Zone. Schering made a donation towards the costs of printing the final report, and to support pharmacist training. The views expressed in this paper are those of the authors.

Competing interests. None identified.

\section{References}

1 Painton D (ed.). The provision of emergency hormonal contraception. London: Royal College of Obstetricians and Gynaecologists Press, 1995

2 Harrison-Woolrych M, Duncan A, Howe J, Smith C. Improving access to emergency contraception. BMJ 2002; 322: 186-187.

3 Ellertson C, Shochet T, Blanchard K, Trussell J. Emergency contraception: a review of the programatic and social science literature. Contraception 2000; 61: 145-186.
4 Department of Health website database of all registered pharmacies and their opening hours. www.nhs.uk

5 Hassell K, Noyce PR, Rogers A. A review of factors that influence the use of community pharmacies and a primary healthcare resource. Int J Pharm Pract 1999; 7: 51-59.

6 www.info.doh.gov.uk/doh/users.nsf

7 Pope C, Mays M (eds). Qualitative research in health care (2nd edn). London: BMJ Books, 2000.

8 A timely service. A Lambeth Southwark and Lewisham HAZ project on access to emergency hormonal contraception via accredited community pharmacies. www.lslhaz.org.uk/options.htm

9 The pill free-for-all. Morning after rules broken by chemists for girl of 15. Daily Mail, 6 January 2001; 7.

10 Blackwell D, Cooper N, Taylor G, Holden K. Pharmacists' concerns and perceived benefits from the deregulation of hormonal emergency contraception. Br J Fam Plann 1999; 25: 100-104.

11 O'Brien KL, Gray NJ. Supplying EHC in Manchester under a group prescribing protocol. Pharm J 2000; 264: 518-519.

12 Ralph SG, Preston AD, Clarke J. Over the counter advice for genital problems: the role of the community pharmacist. Int J STD AIDS 2001; 12: 513-515.

13 Seston EM, Holden K, Cantrill J. Emergency hormonal contraception: the community pharmacy perspective. J Fam Plann Reprod Health Care 2001; 27: 203-208.

14 Wearn A, Gill P, Gray M, Li Wan Po A. Pharmacists views on deregulating emergency hormonal contraception. Pharm J 2001; 266: 89-92. 